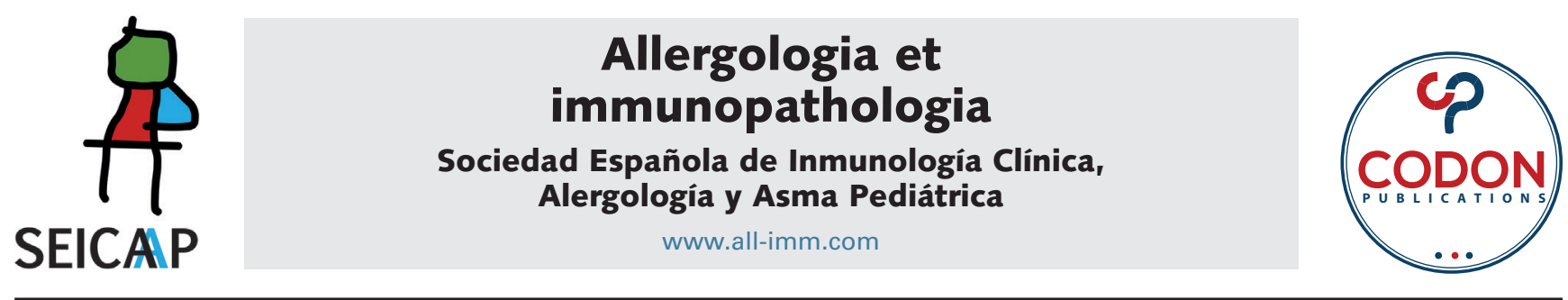

POINT OF VIEW

OPEN ACCESS (c) (1) (2)

\title{
Do gender and puberty influence allergic diseases?
}

\section{Cristine Secco Rosário ${ }^{a *}$, Cristina Alves Cardozob, Herberto José Chong Neto ${ }^{a}$, Nelson Augusto Rosário Filhoa}

aPediatric Allergy and Immunology, Federal University of Parana, Parana, Brazil

${ }^{b}$ Department of Pediatrics, Universidade Positivo, Curitiba, Parana, Brazil

Received 2 June 2020; Accepted 8 September 2020

Available online 1 March 2021

\section{KEYWORDS \\ gender; \\ allergy; \\ rhinitis; \\ sex; \\ puberty}

\begin{abstract}
Differences between biological sex, gender identity, and their impact on health may have significant implications for the prevention, screening, diagnosis, and treatment of several diseases, including allergies. Asthma, allergic rhinitis (AR), atopic dermatitis (AD), and allergic conjunctivitis (AC) have different prevalences and different risk factors in infancy. Although boys present allergies more often in childhood, it quickly changes during girls' sexual development, leading to lifelong female predominance of allergic diseases. This can be explained by the influence of sexual hormones, different lifestyles adopted by men and women, microbiota diversity, diet distinctions, professional options, and adherence to treatment, among others. Gender-related aspects should become essential parameters in allergology to diagnostic and therapeutic stratification, associated with molecular, genetic, and epigenetic patterns. Longitudinal studies would be interesting to evaluate possible mechanisms underlying these differences in prevalence. Sex- and gender-specific observations beyond 14 years of age are scarce and further allergic multimorbidity studies in different populations, especially in adults, are necessary.

(c) 2021 Codon Publications. Published by Codon Publications.
\end{abstract}

*Corresponding author: Cristine Secco Rosário. Rua Padre Camargo, 453. Alto da Gloria, Curitiba - PR - Brazil.

Email address: cristinerosario@hotmail.com 


\section{Allergy and Puberty}

The differences between biological sex, gender identity, and their impact on health can have significant implications for the prevention, screening, diagnosis, and treatment of various diseases, including allergic diseases. Gender and sex are multidimensional, interactive, intertwined, and are sometimes difficult to detach, so the use of the two words (gender and sex) can help to understand the social, cultural, and biological context. ${ }^{1}$

Prevalence of allergic rhinitis (AR) is higher among boys than girls in childhood (0-10 years of age). On the contrary, females display a higher prevalence of AR during adolescence (11-17 years) as compared to their male counterparts. However, when they reach adulthood (18-79 years), there is no difference in prevalence between genders. The same pattern occurs, albeit even more pronounced, for the prevalence of coexisting AR and asthma. ${ }^{2,3}$

In the first year of life, rates of allergic sensitization (specific immunoglobulin $\mathrm{E}$, IgE, production) are significantly higher in males, as are serum levels of total IgE. In this age group, serum levels of total IgE appear to be under a strong genetic influence and may not predict levels of total IgE in the same individual later in life. Increased serum IgE levels and a higher prevalence of sensitization in boys remain until adolescence. After puberty, total serum and allergen-specific IgE antibodies in men are thought to remain higher or comparable to those in women. In adulthood, IgE levels decrease in both genders. In addition to changes during life, IgE levels are also influenced by menstrual periods and pregnancy, suggesting the participation of sex hormones in their regulation. ${ }^{4}$

Although the influence of sex hormones on dendritic cell function, innate immune sensing in the airways, $T$ cell development and activation and $B$ cell responses are well described, it remains unclear how sex hormones directly influence the process of sensitization in the young, adolescents, and adults. There is, however, a well-defined role for sex hormones in modifying allergic symptoms following sensitization. Epidemiologic and experimental studies suggest that female sex hormones, in general, enhance the immunological recall responses, likely leading to exaggerated disease, whereas testosterone dampens the same responses. These effects increase the risk of allergic diseases in adult females; although, paradoxically, females, in general, appear to display both lower levels of IgE and prevalence of sensitization compared to males. ${ }^{4}$

An analysis performed in 4500 Brazilian children aged 13-14 years has shown that females not only had a higher prevalence of AR compared to males but also of allergic rhinoconjunctivitis (ARC), asthma, allergic conjunctivitis $(A C)$, and atopic dermatitis (AD) (Figure 1A). Interestingly, there was an opposite allergic sensitization pattern with respect to sex, with more allergic sensitization in boys than in girls (Figure 1B). Moreover, it has also been observed that monosensitization was more frequent in females, while polysensitization was more common in males. ${ }^{5}$

A global meta-analysis showed sex-related differences in rhinitis prevalence with a switch at around puberty from a male predominance to a female predominance. The authors found no predominance in the prevalence of rhinitis in either adult males or females, although the number of studies was small. In the future, it will be essential to perform longitudinal studies in which follow-up is maintained into adulthood. ${ }^{6}$

A meta-analysis of longitudinal birth cohorts showed a sex shift from a higher incidence in boys before puberty towards a rather sex-balanced incidence after puberty onset. The elevated risk of asthma and rhinitis incidences in teenage girls should lead to more consideration of a sex-specific and age-specific focus on diagnosis and management of respiratory diseases. ${ }^{7}$

The complexity of most allergic diseases is based on a dynamic heterogeneous combination of hyperresponsiveness, dysregulated immune response, chronic inflammation, and tissue remodeling in affected organs. It is vital to systematically investigate sex disparities, possibly in different age groups, allergic disease incidence, and their outcomes. When they are identified, it is necessary to elucidate their biological basis and understand if better outcomes could be obtained with sex-specific treatment modifications. ${ }^{8}$

There are different risk factors for developing allergic diseases in boys and girls. A longitudinal study found that obesity, along with rhinitis and current smoking were risk factors for developing asthma in girls, while the main risk factors for boys were reduced FEV1, seasonal allergic symptoms, and a family history of asthma. ${ }^{9}$ Obesity is known to be associated with chronic low-grade systemic type 1 inflammation. Asthma in obese children has previously been reported to be mainly non-eosinophilic in girls but not in boys, and obesity has been related to neutrophilic airway inflammation in female but not in male adults. Adolescents with recurrent wheezing, both girls and boys, reported more symptoms of rhinitis and parental-reported allergic symptoms at baseline, as compared to patients who had never wheezed. The association between wheezing and rhinitis in adolescents has been previously described, but further studies are necessary to fully establish the relationship between weight and respiratory symptoms, especially regarding sex differences. ${ }^{9}$

To optimize clinical practice, it is necessary to understand, in addition to the molecular mechanisms and biomarkers, the phenotypes of allergic diseases, as well as the differences in their distribution between sexes. This is recognized as an innovative element, as there is scientific evidence that men and women not only have distinct clinical manifestations for the same disease but also have different therapeutic responses. These can be influenced by biological (hormonal, organic) and socio-cultural factors (adherence to treatment, work, purchasing power). ${ }^{8}$

Men and women have different lifestyles, in terms of choosing specific professional careers, sports, intake of hormonal medications, and quality of diet. Immune cells (lymphocytes, monocytes, eosinophils, and mast cells) express hormone receptors and, therefore, may be highly influenced by endogenous and exogenous hormones, which fluctuate in women. ${ }^{1}$

Longitudinal studies would be valuable to raise possible mechanisms underlying these differences in prevalence. Sex- and gender-specific evaluations beyond 14 years of age are scarce and further allergic multimorbidity studies in different populations, especially in adults, are necessary. ${ }^{3}$ 

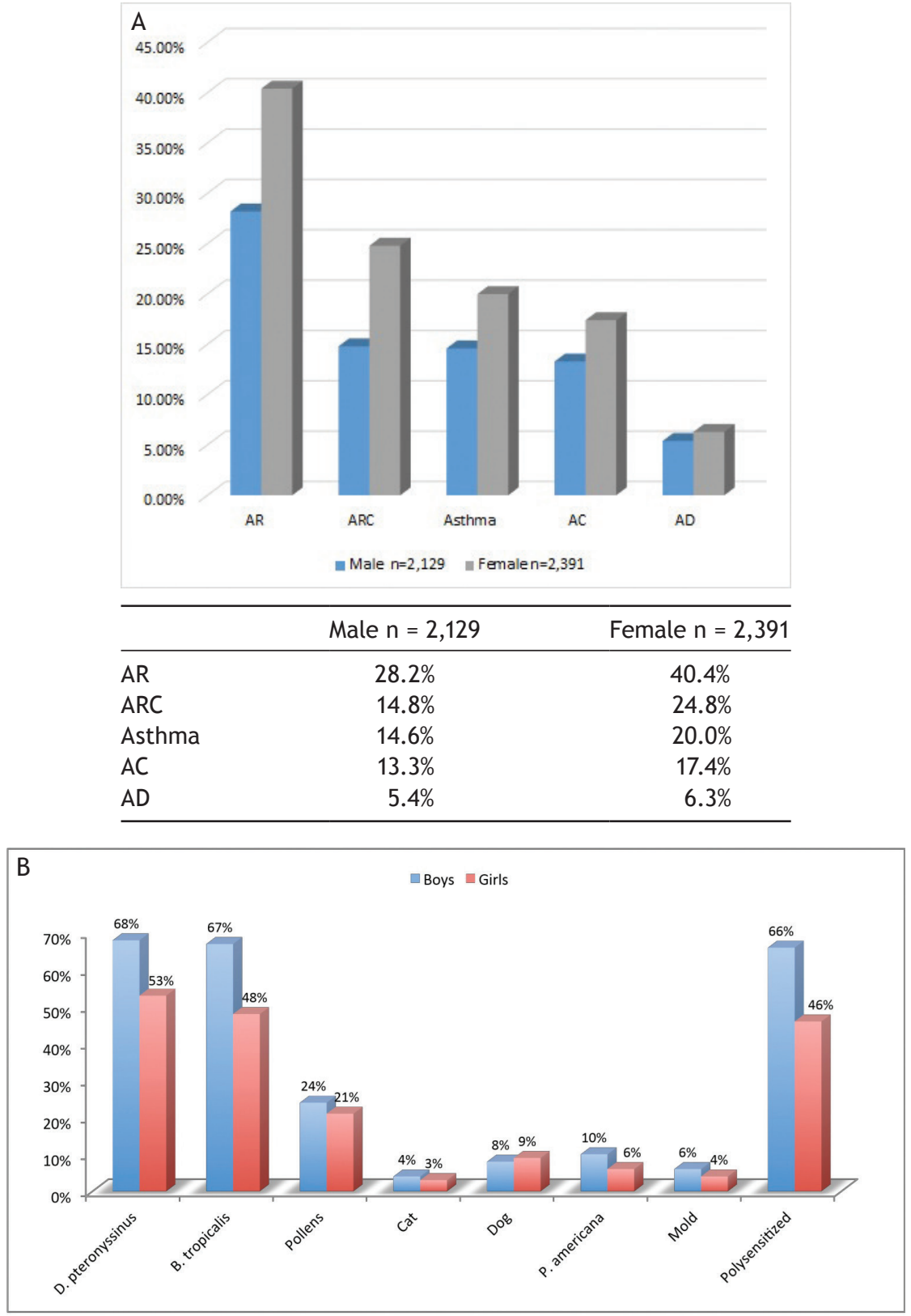

\begin{tabular}{lcccccccc}
\hline & D. & B. & & \multicolumn{5}{c}{ P. } \\
& pteronyssinus & tropicalis & Pollens & Cat & Dog & americana & Mold & Polysensitized \\
\hline Boys & $68 \%$ & $67 \%$ & $24 \%$ & $4 \%$ & $8 \%$ & $10 \%$ & $6 \%$ & $66 \%$ \\
Girls & $53 \%$ & $48 \%$ & $21 \%$ & $3 \%$ & $9 \%$ & $6 \%$ & $4 \%$ & $46 \%$ \\
\hline
\end{tabular}

Figure 1 (A) Prevalence of allergic diseases according to gender, $n=4520$. (B) Allergic sensitization pattern according to gender, $n=472$. AR: allergic rhinitis; ARC: allergic rhinoconjunctivitis; AC: allergic conjunctivitis; AD: atopic dermatitis.

* Adapted from reference 7 with permission $(n=472)$.

\section{Conflicts of interest}

All the authors have nothing to disclose related to this article. The content of the manuscript has not been published or submitted for publication elsewhere.

We confirm that this work is original and has not been published elsewhere. All authors have conceived and planned the project, as well as analyzed and interpreted the data. We all have organized and critically reviewed the manuscript's content and approved the final content.

\section{References}

1. Franconi F, Campesi I, Colombo D, Antonini P. Sex-gender variable: methodological recommendations for increasing 
scientific value of clinical studies. Cells. 2019;8(5):476. https:// doi.org/10.3390/cells8050476

2. Keller T, Hohmann C, Standi M, Wijga AH, Gehring U, Melén E, et al. The sex-shift in single disease and multimorbid asthma and rhinitis during puberty - a study by MeDALL. Allergy. 2018;73(3):602-614. https://doi.org/10.1111/all.13312

3. Frohlich M, Pinart Gilberga M, Keller T, Reich A, Cabieses B, Hohmann C, et al. Is there a sex-shift in prevalence of allergic rhinitis and comorbid asthma from childhood to adulthood? A meta-analysis. Clin Transl Allergy. 2017;7:44. https://doi. org/10.1186/s13601-017-0176-5

4. Leffler J, Stumbles PA, Strickland DH. Immunological processes driving lgE sensitisation and disease development in males and females. Int J Mol Sci. 2018;19:1554. https://doi. org/10.3390/ijms19061554

5. Rosario CS. Fatores associados à conjuntivite alérgica em adolescents de Curitiba, Paraná. [Dissertação] 2018. Curitiba (PR): Universidade Federal do Paraná. https://hdl.handle. net/1884/65989 (2018). Accessed 14 Apr 2020.
6. Pinart $M$, Keller $T$, Reich $A$, Fröhlich $M$, Cabieses $B$, Hohmann $C$, et al. Sex-related allergic rhinitis prevalence switch from childhood to adulthood: a systematic review and meta-analysis. Int Arch Allergy Immunol. 2017;172(4):224-235. https://doi.org/10.1159/000464324

7. Hohmann C, Keller T, Gehring U, Wijga A, Standi M, Kull I, et al. Sex-specific incidence of asthma, rhinitis and respiratory multimorbidity before and after puberty onset: individual participant meta-analysis of five birth cohorts collaborating in MeDALL. BMJ Open Respir Res. 2019;6(1):e000460. https:// doi.org/10.1136/bmjresp-2019-000460

8. De Martinis M, Sirufo MM, Suppa M, Di Silvestre D, Ginaldi L. Sex and gender aspects for patient stratification in allergy prevention and treatment. Int J Mol Sci. 2020;21(4):1535. https://doi.org/10.1016/j.jaip.2018.08.008

9. Kalm-Stephens P, Nordvall L, Janson C, Neuman A, Malinovschi A, Alving K. Different baseline characteristics are associated with incident wheeze in female and male adolescents. Acta Paediatr. 2020 Mar 18 [epub ahead of print]. https://doi.org/10.1111/apa.15263 